\title{
Analysis in the heat transfer process of water in a bathtub with and without bubbles
}

\author{
Jidong $\mathrm{Li}^{1, \mathrm{a}}$ \\ ${ }^{1}$ North China Electric Power University, Baoding 071000, China; \\ a759636597@qq.com
}

\begin{abstract}
Keywords: 3D Heat-Conduction Differential Equations, Finite Element Analysis, Equivalent Heat Conductivity, Numerical Simulation.
\end{abstract}

Abstract. This paper proposed a basic model and an optimized model to obtain the temperature field of water in a bathtub with and without bubbles. Firstly, the basic heat transfer model is established based on the three-dimensional N-S equations, the heat transfer differential equations and cuboid boundary conditions. Furthermore, the optimal model, which takes the influence of bubbles into account, is developed. The effect is equivalently transferred to modify the heat transfer coefficient. The result shows that the bubbles will generally reduce the heat transfer efficiency. In addition, the result is carried out by means of finite element analysis, using the software Fluent.

\section{Introduction}

There are some previous researches on establishing the 3-D unsteady mathematical model to solve the heat transfer problem. Based on Douglas ADI method, three dimensional differential equations are utilized to simulate the heat process and optimize the control of heating furnace[1]. In order to achieve the control of suitable temperature, Professor Zhou built an inverse problem solution model for three-dimensional transient temperature to get the optimum solution of nonlinear inverse problem[2]. Most of the these references contribute a lot to the problem we are analyzing. Nevertheless, little has been devoted to the heat transfer in bathtub., not to mention the influence of the bubbles.

\section{Basic Model}

\subsection{Analysis of The Problem}

At the beginning, the shape of the bathtub is designed as a simple cuboid whose length, width and height are respectively $1.8 \mathrm{~m}, 0.7 \mathrm{~m}$ and $0.8 \mathrm{~m}$. Then a three-dimensional rectangular coordinate is built on the bathtub. Next carry a micro-control volume out of the cuboid as the object of the research.

According to the law of energy conservation, three-dimensional differential equations for heat transfer can be established based on the boundary conditions [3].

\subsection{Establishing the Model}

It is hypothesized that the water in the bathtub is incompressible fluid, namely $\rho=$ const . Then the continuity equation and the energy differential equation can be built as follows:

$$
\begin{gathered}
\frac{\partial u}{\partial x}+\frac{\partial v}{\partial y}+\frac{\partial w}{\partial z}=0 \\
\frac{\partial t}{\partial \tau}+u \frac{\partial t}{\partial x}+v \frac{\partial t}{\partial y}+w \frac{\partial t}{\partial z}=a\left(\frac{\partial^{2} t}{\partial x^{2}}+\frac{\partial^{2} t}{\partial y^{2}}+\frac{\partial^{2} t}{\partial z^{2}}\right)
\end{gathered}
$$

Where $t$ respects temperature and $\tau$ means time.

Where $a=\frac{\lambda}{\rho c}$ indicating the thermal diffusivity of the water.

Next, the three-dimensional N-S equations are built as follows: 


$$
\left\{\begin{array}{l}
\frac{d u}{d \tau}=-\frac{1}{\rho} \frac{\partial p}{\partial x}+v\left(\frac{\partial^{2} u}{\partial x^{2}}+\frac{\partial^{2} u}{\partial y^{2}}+\frac{\partial^{2} u}{\partial z^{2}}\right) \\
\frac{d v}{d \tau}=-\frac{1}{\rho} \frac{\partial p}{\partial y}+v\left(\frac{\partial^{2} v}{\partial x^{2}}+\frac{\partial^{2} v}{\partial y^{2}}+\frac{\partial^{2} v}{\partial z^{2}}\right) \\
\frac{d w}{d \tau}=-g-\frac{1}{\rho} \frac{\partial p}{\partial z}+v\left(\frac{\partial^{2} w}{\partial x^{2}}+\frac{\partial^{2} w}{\partial y^{2}}+\frac{\partial^{2} w}{\partial z^{2}}\right)
\end{array}\right.
$$

Where $u, v$ and $w$ respectively indicates the velocity in direction $x, y$ and $z, p$ represents the pressure and $g$ means the gravitational acceleration.

In terms of the heat transfer process of water, the partial differential equations are built as follows:

$$
\left\{\begin{array}{l}
h_{x}=-\left.\lambda \frac{\partial t}{\partial x} \cdot \frac{1}{t_{w}-t}\right|_{x=0} \\
h_{y}=-\left.\lambda \frac{\partial t}{\partial y} \cdot \frac{1}{t_{w}-t}\right|_{y=0} \\
h_{z}=-\left.\lambda \frac{\partial t}{\partial z} \cdot \frac{1}{t_{w}-t}\right|_{z=0} \\
h_{z}=-\left.\lambda \frac{\partial t}{\partial z} \cdot \frac{1}{t_{w}-t}\right|_{z=H}
\end{array}\right.
$$

Where $h_{x}, h_{y}, h_{z}$ and $h_{z}$ respectively represents the heat transfer coefficient of the front, left, top and bottom sides.

In the heating process, the change of the temperature field with the passage of time is presented in the figure below:
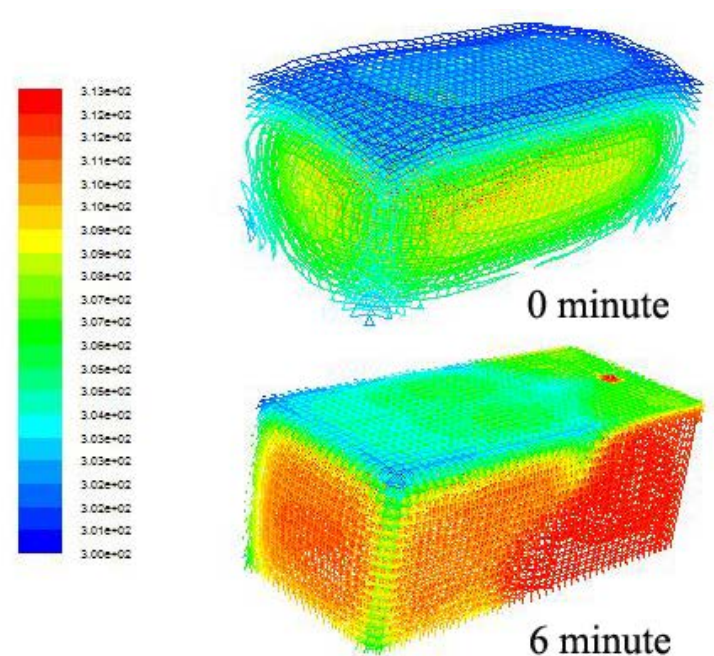

6 minute
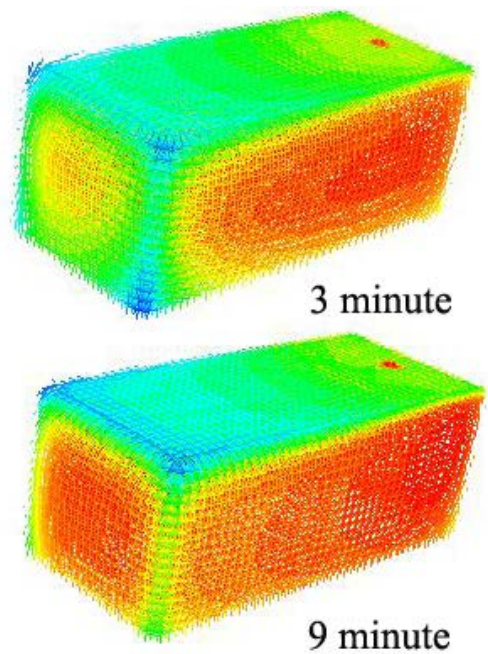

Fig 1 the change of the temperature field of water in heating process

\section{Modified Model}

The water and the air do not contact directly with a layer of bubble between them on the top surface, which makes the heat transfer coefficient smaller and reduce the efficiency of the heat transfer.

Based on the component of the bubble, some basic figures can be obtained in reference to the sheet of the physical property parameter. According to the sheet of surface tension of common materials, we obtain the surface tension of the bubble which is expressed as $\sigma=2.70 \mathrm{dyn} / \mathrm{cm}=22.7 \times 10^{-3} \mathrm{~N} / \mathrm{m}$. The pressure difference between inner and outer is expressed as $\Delta p=41.27 \mathrm{~Pa}$.

The equation of the surface tension and the bubble's radius is expressed as below:

$$
\Delta p=2 \sigma / R
$$


Then we can obtain the size of the bubble: $R=2 \sigma / \Delta p \approx 11 \mathrm{~mm}$

Before analyzing the problem, some reasonable assumptions are made as follows:

- The thickness of the bubble layer is $3 \mathrm{~mm}$.

- The bubbles are in uniform distribution on the water.

- The arrangement of bubbles accords with a regular rule that particles accumulate in a hexagonal close-packed form.

As shown in the figure above, the width of the air interval is (3-2d) $\mathrm{mm}$.

We introduce a equivalent heat conductivity coefficient $\lambda_{b}$, an equation of the equivalent value and the heat transfer coefficient of air and water is built as below:

$$
\frac{\lambda_{b}}{3 \times 10^{-3}}=\frac{\lambda_{\text {air }}}{2 d}+\frac{\lambda_{\text {water }}}{3 \times 10^{-3}-2 d}
$$

Then the equivalent heat conductivity coefficient is obtained.

Neglecting the temperature distribution inside the bubble, we establish the equation of heat flow under the circumstances with and without the layer of bubble. It is presented as below:

$$
q=\frac{t-t_{f}}{\frac{\delta}{\lambda}+\frac{1}{h}}=\frac{t-t_{f}}{\frac{1}{h^{\prime}}}
$$

Where $h$ is the heat transfer coefficient without bubbles.

$h$ ' is the heat transfer coefficient with bubbles, namely the equivalent heat transfer coefficient considering the influence of bubbles.

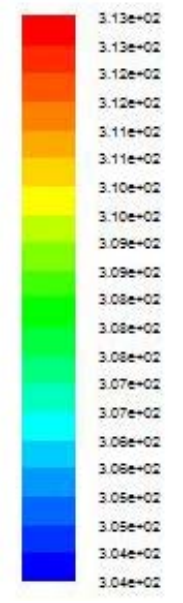

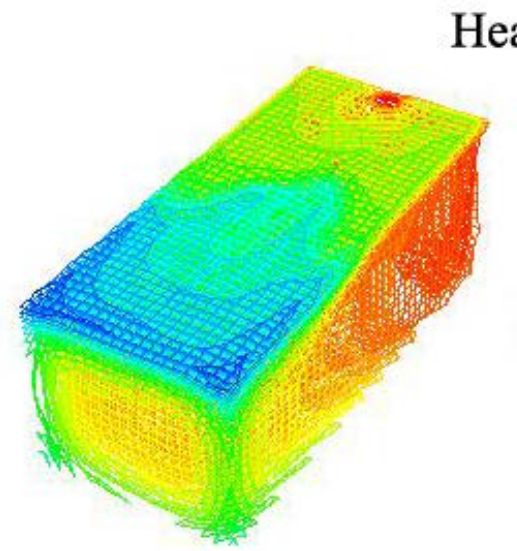

Without Bubbles
Heating Process

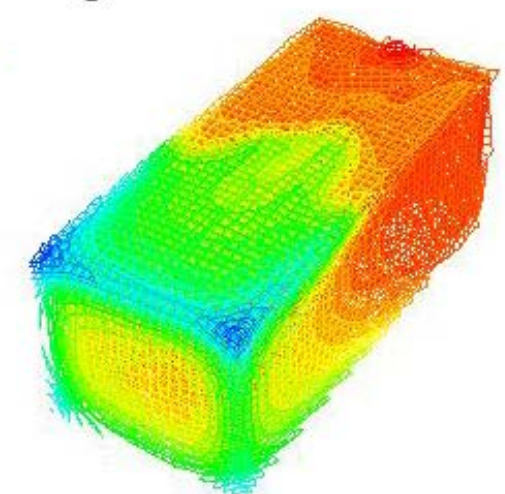

With Bubbles

Fig 2 the temperature fields of water with and without bubbles

As shown in the figure above, we can see that the rate and efficiency of heat transfer is obviously reduced, which accords with our analysis.

\section{Summary}

Section one of our paper develops a basic heat transfer model based on the three-dimensional N-S equations and the heat transfer differential equations. By calculating the temperature field. In section two, we calculate the equivalent heat transfer coefficient with bubbles and the result demonstrates that the heat transfer efficiency is reduced due to bubbles.

\section{References}

[1] Yuan Wenqing, Chen Xiao, Hu Mingjuan, "Computer Simulation of Three Dimensional Unsteady Temperature Fields during Heating-up Process”, Transactions of Metal Heat Treatment, vol.12,No.2,pp.35-42,June 1991.

[2] Zhou Wei, Wang Xiaochan, "Unsteady Temperature Simulation under Variable Boundary Conditions for Venlo Type Conditions”, Transaction of the Chinese Society of Agricultural 
Machinery, vol.45, No11, ,pp.305-307, November, 2014.

[3] Zhang Jinxia, 2014, "The Simulation Analysis of the Influence for Swimming Pool Water Temperature Field in Different Heating Modes”,PhD diss., Yanshan University., 2014. 